(C) <2019>. This manuscript version is made available under the CC-BY-NC-ND 4.0 license http://creativecommons.org/licenses/by-nc-nd/4.0/

The definitive publisher version is available online at https://doi.org/10.1016/j.asoc.2019.105528 


\title{
A DEEP NEURAL NETWORK BASED CLASSIFIER FOR BRAIN TUMOR DIAGNOSIS
}

\author{
Ambeshwar Kumar \\ School of Computing, SASTRA Deemed University, \\ Thanjavur, Tamilnadu, 613401, India. \\ Email ID: ambeshwar.kumar@gmail.com \\ Manikandan Ramachandran \\ School of Computing, SASTRA Deemed University, \\ Thanjavur, Tamilnadu, 613401, India. \\ Email ID: srmanimt75@gmail.com
}

\begin{abstract}
Amir H. Gandomi
Corresponding Author

School of Business, Stevens Institute of Technology, Hoboken, NJ 07030, USA

Faculty of Engineering and IT, University of Technology Sydney, Ultimo, Australia

Email ID: a.h.gandomi@gmail.com
\end{abstract}

\section{Rizwan Patan}

School of computing Sciences and Engineering, Galgotias University, Gr. Noida, Uttar Pradesh, 201308, India

Email ID: prizwan5@gmail.com

\section{Szymon Lukasik}

Faculty of Physics and Applied Computer Science, AGH University of Science and Technology, Poland Systems Research Institute, Polish Academy of Sciences, Poland

Email ID: slukasik@agh.edu.pl

Ravichandran Kattur Soundarapandian

School of Computing, SASTRA Deemed University,

Thanjavur, Tamilnadu, 613401, India

Email ID: raviks@it.sastra.edu 


\begin{abstract}
Classification process plays a key role in diagnosing brain tumors. Earlier research works are intended for identifying brain tumors using different classification techniques. However, the False Alarm Rates (FARs) of existing classification techniques are high. To improve the early-stage brain tumor diagnosis via classification the Weighted Correlation Feature Selection Based Iterative Bayesian Multivariate Deep Neural Learning (WCFSIBMDNL) technique is proposed in this work. The WCFS-IBMDNL algorithm considers medical dataset for classifying the brain tumor diagnosis at an early stage. At first, the WCFSIBMDNL technique performs Weighted Correlation-Based Feature Selection (WC-FS) by selecting subsets of medical features that are relevant for classification of brain tumors. After completing the feature selection process, the WCFS-IBMDNL technique uses Iterative Bayesian Multivariate Deep Neural Network (IBMDNN) classifier for reducing the misclassification error rate of brain tumor identification. The WCFS-IBMDNL Technique was evaluated in JAVA language using Disease Diagnosis Rate (DDR), Disease Diagnosis Time (DDT), and FAR parameter through the epileptic seizure recognition dataset.
\end{abstract}

Keywords: Deep Neural Network, Bayesian Multivariate Linear Regression, Brain Tumor, Feature selection, Iteratively Reweighted Least Squares, Least absolute deviations, Medical Features, Weighted Correlation 


\section{INTRODUCTION}

Early diagnosis and treatment of brain tumors are imperative to prevent permanent damage to the brain or death of the patient. At the level of medical data analysis, the features election and classification process are the ones intensively used to identify the patient data whether it is normal or abnormal. The indefinite and uncontrollable proliferation of cells causes tumors in the brain. Brain tumors impact people of all ages. Feature selection is a preprocessing step in disease diagnosis for reducing the complexities in the diagnostic model. A tumor is a mass of tissue composed of abnormal cells. Several research tools have been developed and are used to diagnose brain tumors with the aid of diverse classification techniques. However, current brain tumor diagnosis approaches perform inadequately in the context of not being able to overcome classification error rates. To address this deficiency, we developed the Weighted Correlation Feature Selection Based Iterative Bayesian Multivariate Deep Neural Learning (WCFS-IBMDNL) technique. WCFS-IBMDNL Technique is developed with weighted correlation-based feature selection and iterative Bayesian multivariate deep neural network classifier.

A diverse set of algorithms were already proposed for the brain tumor diagnosis, for instance, an ensemble classifier [1]. The ensemble classification with bagging and decision tree outperforms the diseases diagnosis for an imbalanced data set. The designed algorithm contained Maxi-mum Relevance and Minimum Redundancy filter to establish the feature selection. However, the diagnosis rate of this classifier was not improved since the generation of diagnostic decision rule was not efficient. Support Vector Machine (SVM) classifier was also usedfor efficient identification of epileptic seizures [2]. However, because of the False Alarm Rate (FAR), the diagnosis time was in this case longer. A Two-hybrid machine learning model's genetic algorithm with SVM and artificial neural network were likewise developed for improving the brain tumor classification accuracy [3]. However, the models failed to minimize the computational complexity in the tumor classification. An Incremental Gaussian Discriminant Analysis was developed for diagnosing the brain tumors [4]. But, the algorithm was not minimizing the false acceptance rate. A supervised machine learning approach was developed for enhancing the performance of whole-brain seizure discovery [5]; however, the diagnosis rate of brain tumors was not adequate. Pattern-and network-based classification techniques were designed for more accurate predictions of brain tumors [6]. In this case, the processing time of this technique was found to be very high. Similarly, molecular approaches have been used to diagnose brain tumors [7]. Logistic model trees were used to detect the presence of epileptic seizures based on EEG signals [8]; however, the diagnosis rate was lower. A novel method was developed for finding significant brain tumor features for disease classification [9] but the time and space complexity during the brain tumors diagnosis was not solved. Gray Level Co-occurrence Matrix (GLCM) and Gray-Level Run-Length Matrix (GRLM) were also developed for classification of brain tumor detection [10]; however, feature selection performance was not effective. An Artificial Bee Colony (ABC) algorithm was used for medical data classification [11] still the accurate disease diagnosis was not attained.

A fuzzy rule-based seizure detection system was developed using the knowledge acquired from experts' reasoning [13]. It does not address the false positive rate of seizure detection. A novel seizure detection algorithm was likewise designed to attain higher sensitivity and a lower false positive rate for diagnosis of brain tumors [14]. Finally, a deep learning-based approach was developed for learning EEG features and predicting epileptic seizures [15]; however, the time complexity of epileptic seizure detection was greater than the proposed WCFS-IBMDNL technique.

Multi-scale radial basis functions (MRBF), modified particle swarm optimization (MPSO) and SVM classifier (MRBF-MPSO-SVM) werecombinedin [16] for identifying the brain tumor disease. However, the time consumption was not minimized. A survey of different 
Computer-Aided Diagnosis (CAD) techniques was introduced for neurological diseases diagnosis in [17]. Fuzzy entropy (FuzzyEn) and distribution entropy (DistEn) were developed in [18] for executing brain tumor disease diagnosis with higher accuracy without minimizing the time consumption. A novel hybrid method called Support Vector Machine (SVM) and Procrustes Analysis (PA) (SVM-PA), was developed in [19] to perform feature selection in the medical database. However, the space complexity was improved since it unable to select the relevant features for disease diagnosis. Online Feature Selection algorithm was presented in [20] based on the Dependency in K nearest neighbors for high-dimensional class-imbalanced data. However, feature selection was not performed effectively. A hybridization approach was introduced in [21] to improve diagnostic accuracy based on the extracted significant diabetes features. However, the space complexity of disease diagnosis was not considered. SVM was designed in [22] for feature selection and detecting diseases without selecting the most relevant features. A hybrid decision support system was introduced in [23] to achieve higher classification performance in diabetes disease diagnosis by extracting relevant features. However, the feature selection accuracy was not adequate. A review of diverse brain tumor diagnostic techniques was previously published in [12].

We have identified, from the analysis of the above literature, that existing methods are affected by several issues such as classification error, not sufficient diagnosis rate, large diagnosis time, not adequate feature selection performance, time and space complexity. To solve the above-said issues, the WCFS-IBMDNL technique is developed for performing brain tumor diagnosis in the medical dataset. The proposed WCFS-IBMDNL technique is implemented with the aid of weighted correlation feature selection and iterative Bayesian multivariate deep neural learning. The correlation-based feature selection discovers more relevant features and Bayesian multivariate deep neural learning deeply examines the selected feature for brain tumor classification. This advantages to increases the DDR and reduces the DDT with FAR in WCFS-IBMDNL technique. The objective of the proposed WCFSIBMDNL Technique is to increase the performance of brain tumor disease diagnosis at an earlier stage with higher DDR.

The major contributions of the WCFS-IBMDNL areas follows:

* First it is proposed to enhance the performance of brain tumor prediction via a classification technique. The proposed WCFS-IBMDNL technique is designed with the implementation of WC-FS and IBMDNN classifier.

* WC-FS is used to execute the feature selection process for performing effective brain tumor detection diagnosis. The novelty of WCFS-IBMDNL is to apply the Pearson correlation coefficient in WC-FS. Pearson correlation coefficient is applied to identify the correlation between two medical attributes for choosing a subset of medical features that are most relevant to the brain tumor disease classification process. A weight value is allocated to all attributes where higher weight attributes are selected for classification and lower weight attributes are removed from the database. This increases the feature selection performance with minimum time and space complexity.

* IBMDNN Classifier is introduced in the proposed WCFS-IBMDNL technique to improve the classification performance of brain tumor diagnosis. Besides, BMLRis used in IBMDNN classifier for deeply analyzing the medical features for classifying whether the patient as normal or abnormal. After the classification, the least absolute error is computed. Finally, the IRLS method is used to minimize the error rate. This helps to increase the disease diagnosis rate with minimizing the FAR.

The structure of the paper is as follows: Section 2 explains the WCFS-IBMDNL technique with the help of an architecture diagram. In Section 3, simulation settings are described and experimental results are discussed in Section 4. Section 5 presents the conclusions of this paper. 


\section{WCFS-IBMDNL}

Recently, many research works are designed to classify the brain tumor features for diagnosing the diseases. But, the correlation among the brain tumor features in the big data set was unable to be performed thus reduces the diseases diagnosis performance. Besides, misclassification results are occurred in traditional methods while performing classification. In order to overcome the above-said issues, WCFS-IBMDNL technique is designed for performing disease diagnosis more accurately.

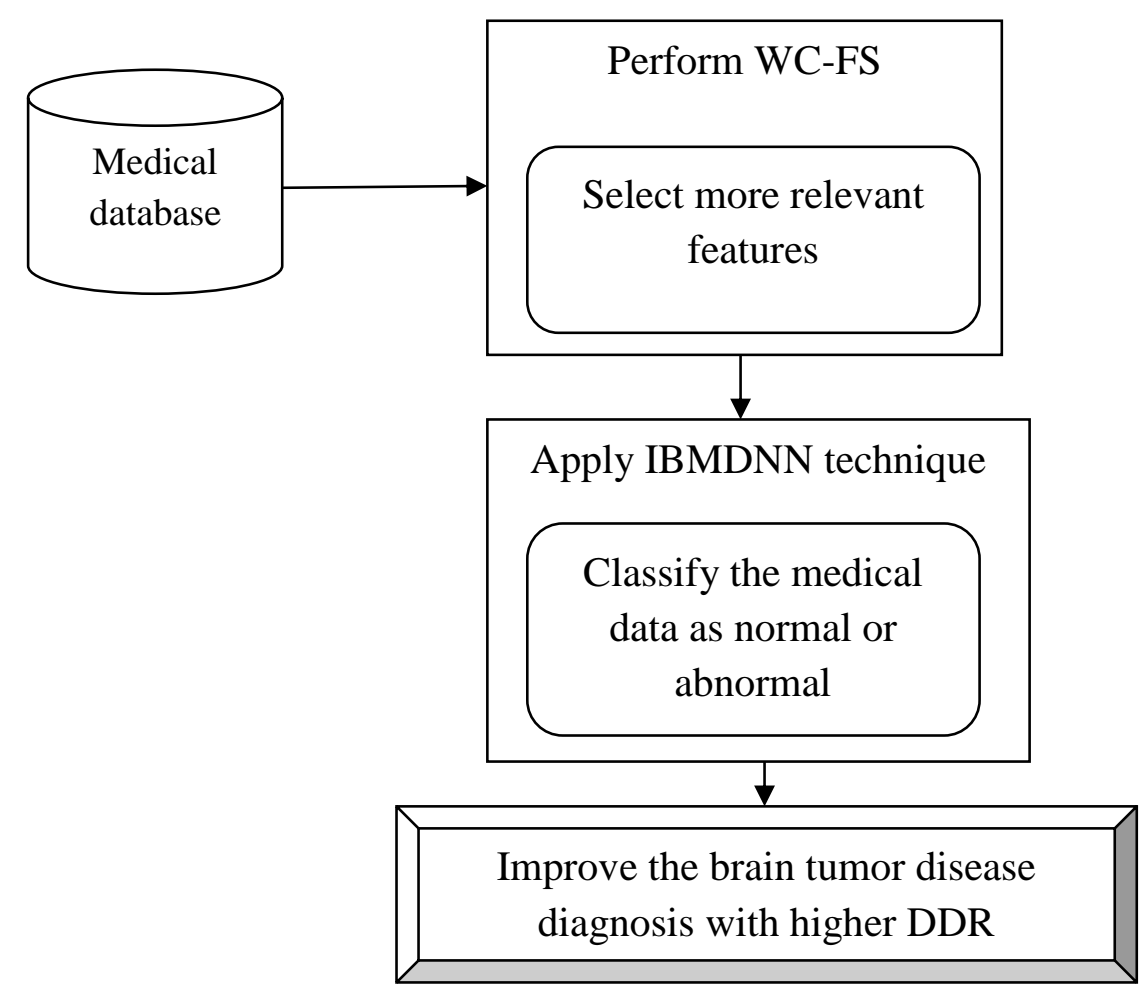

Figure 1 Architecture of proposed WCFS-IBMDNL technique

The WCFS-IBMDNL Technique was developed to improve the performance of brain tumor diagnosis with minimal time requirements. The WCFS-IBMDNL techniquecombines the WC-FS and the IBMDNN classifier. The WCFS-IBMDNL techniqueincludes two main processes: subset feature selection and classification in Figure 1. In contrast to existing approaches, the weighted correlation method is usedinthe WCFS-IBMDNL techniqueto choose medical features that are relevant for brain tumor classification. After choosing a subset of features, the WCFS-IBMDNL techniqueapplies the IBMDNN classifier, foreffective brain tumor diagnosis with minimal time complexity. The WCFS-IBMDNL techniquesis presented in details below.

\subsection{Weighted Correlation-based Feature Selection}

Feature selection plays a significant role in brain tumor diagnosis. The traditional method performs feature selection process for classifying the diseases in the medical database. However, during the feature selection, the redundant features are unable to be eliminated since the relationship among the features not measured which increases the time and space complexities in disease diagnosis. In addition, backward search methods are employed to identify the novel interaction among the features. However, the redundant subset elimination 
is less efficient. Therefore, WC-FS is performedwithin WCFS-IBMDNL technique to reduce the space and time complexity. The weighted correlation model is usedin the WCFS-IBMDNL technique. It employs the weight value of each medical feature in a given medical dataset to select the subset of medical features in a minimal amount of time. Correlation values, which describe the degree to which medical features are related, range between 1 and-1. Closely relatedvalues with either a positive or negative correlation are characterizedbya correlation close to 1 or -1 , respectively. The Pearson correlation coefficient is used in WC-FS to conclusively determine the correlation between two medical attributes in an input dataset. After this, WC-FS applies a weight to each medical attribute in an input medical dataset according to their measured correlation values. This weight value helps WC-FS find relevant medical features for prediction of brain tumorsin less time as compared to state-of-the-art approaches. The medical features with higher weight values are consideredasthe mostsignificant features for brain tumor classification. Thus, WC-FS selectsthemedical features that have higher weight valuesand subsequently remove the medical features with lower weight values.

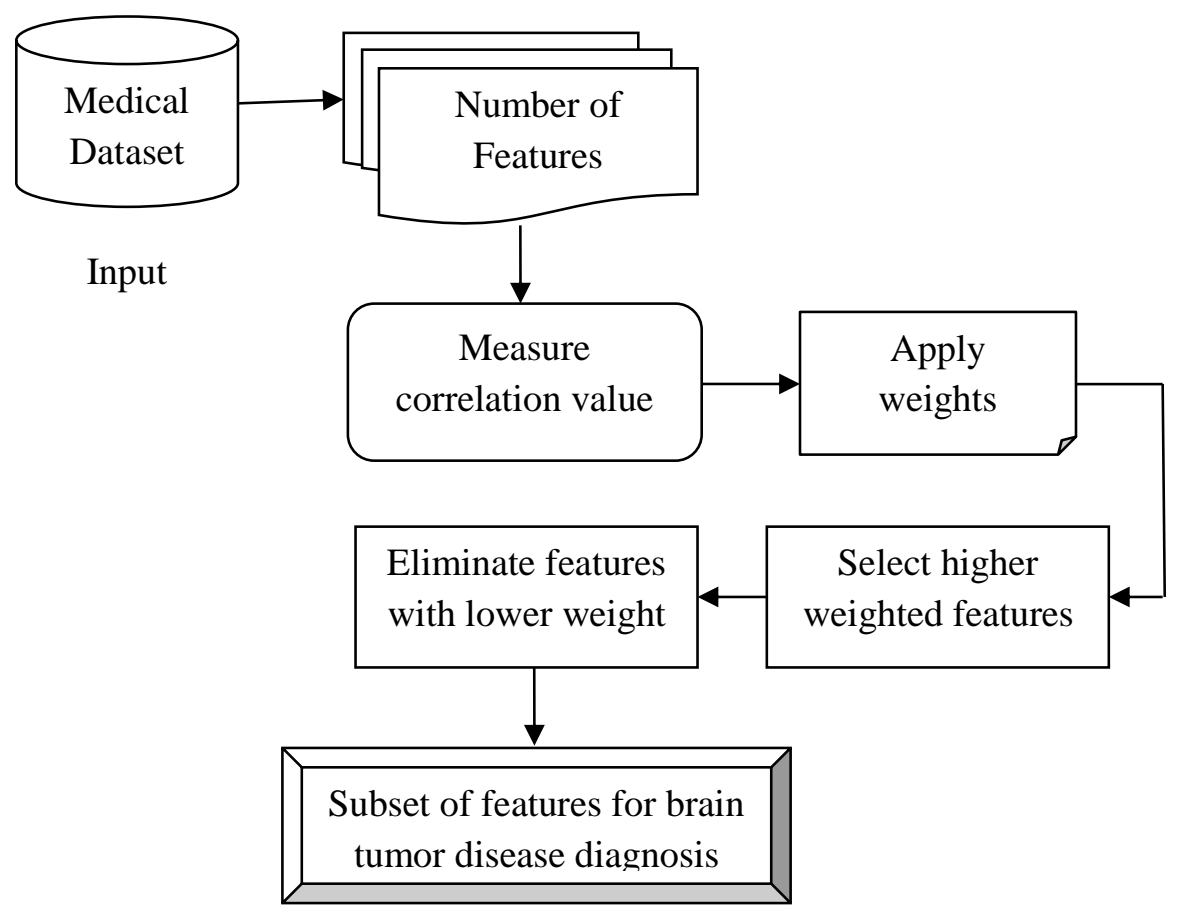

Figure 2: Processes of Weighted Correlation-based Feature Selection

In figure 2showsthe flow process of WC-FS within the WCFS-IBMDNL technique. As depicted in figure 2, WC-FS first takes a medical dataset (i.e., the Epileptic Seizure Recognition Dataset) as input. Then, WC-FS estimates the correlation between the medical features with supportfrom thePearson correlation coefficient. Next, WC-FS assigns a weight toeach medical feature in a given medical dataset and selects the higher weighted medical features as a subset of medical features for prediction of brain tumors.

Let us consider the input medical dataset ' $M D$ ' that contains several features represented as ' $F_{i}=F_{1}, F_{2}, \ldots F_{n}$ ' where $i=1,2, \ldots . n$. Here, ' $n$ ' indicates the total number of medical features in a given dataset. The WC-FS computes the degree of correlation ' $c_{i}$ ' between twomedical features ' $F_{1}$ ' and ' $F_{2}$ ' using the equation below. 


$$
c_{i}=\frac{n \sum F_{1} F_{2}-\sum F_{1} \sum F_{2}}{\sqrt{n\left(\sum F_{1}{ }^{2}-\left(\sum F_{1}\right)^{2}\right)\left(n \sum F_{2}^{2}-\left(\sum F_{2}\right)^{2}\right)}}
$$

In equation (1), ' $n$ 'indicates the number of medical features in the medical dataset. Next, WC-FS determines the weight ' $\vartheta_{i}$ ' based onthe correlation value ' $c_{i}$ ' for each medical feature using the equation below.

$$
\vartheta_{i} \rightarrow \sum_{i=1}^{n} F_{i}
$$

By means of equation (2), WC-FS introduces weight ' $\vartheta_{i}$ ' of each medical feature. Next, WC-FS selects the higher weighted medical features using the equation below.

$$
F^{*}=\arg \max \vartheta_{i}\left\{F_{1}, F_{2}, \ldots F_{n}\right\}
$$

In equation (3), ' $F^{*}$, represent the chosen subset of features. WC-FS uses equation (3) to pick the related subset of medical features for brain tumor classification, which eliminates irrelevant medical features. As a result, the WCFS-IBMDNL technique significantly enhances the performance of feature selection as compared to conventional approaches. The algorithmic process of WC-FS is shown below.

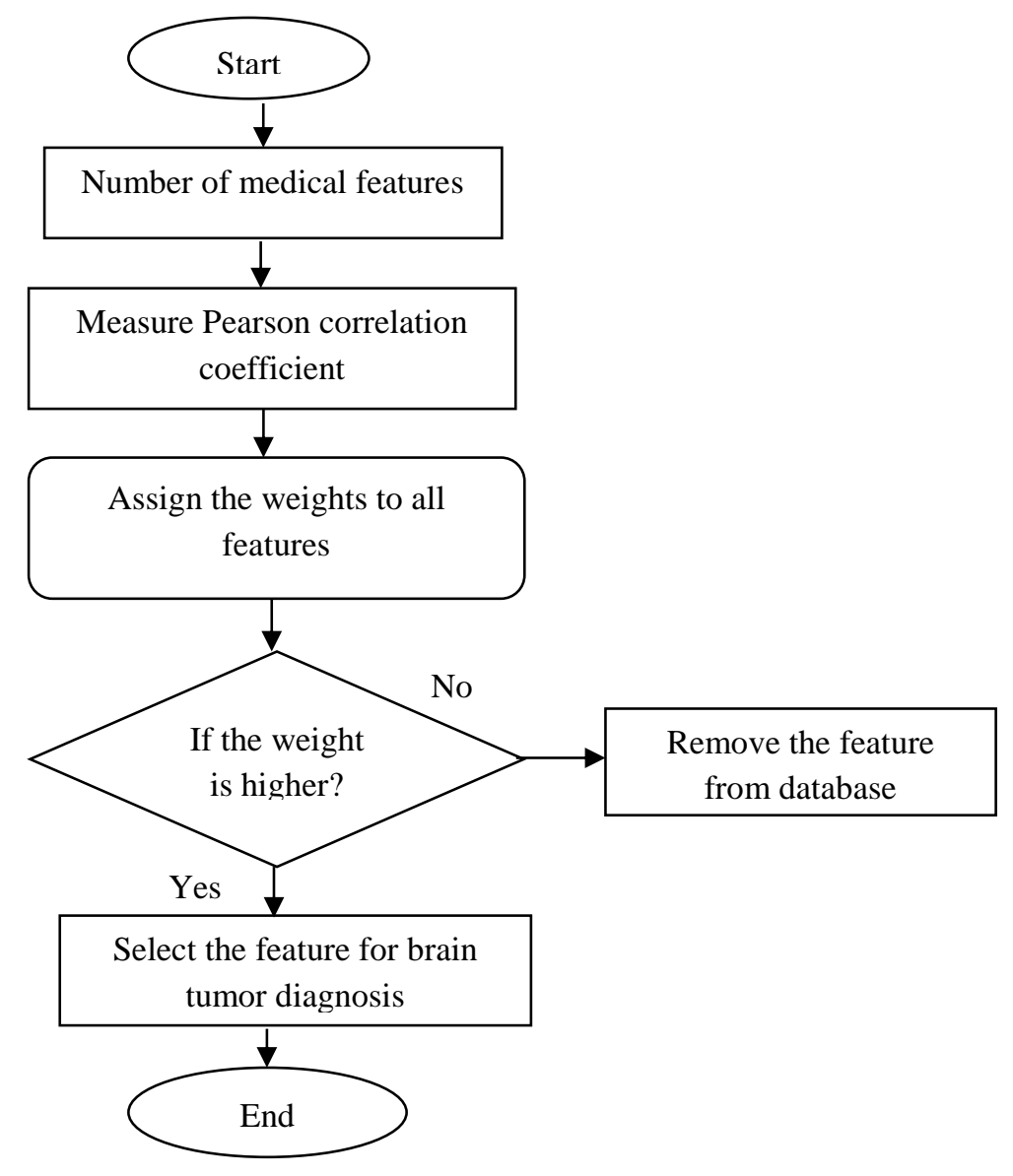

Figure 3 Flow process of feature selection using WC-FS 
As shown in figure 3, the WC-FS process is applied to choose the relevant features for brain tumor disease diagnosis. At first, the medical dataset is considered as input. From that, the Pearson correlation coefficient is applied to the input dataset to calculate the correlation between two medical features. It provides the correlation value of +1 and -1 where the higher correlation represents the +1 and lower correlation represents -1 . Depending upon the correlation measure, the weights are allocated to each medical feature. Then the higher weight features are said to be more relevant which is further considered for the classification process. Besides, the lower weight features are redundant and thus they removed from the database. This in turns, space and time complexity of brain tumor diseases diagnosis is highly reduced.

Algorithm 1.Weighted Correlation-based Feature Selection

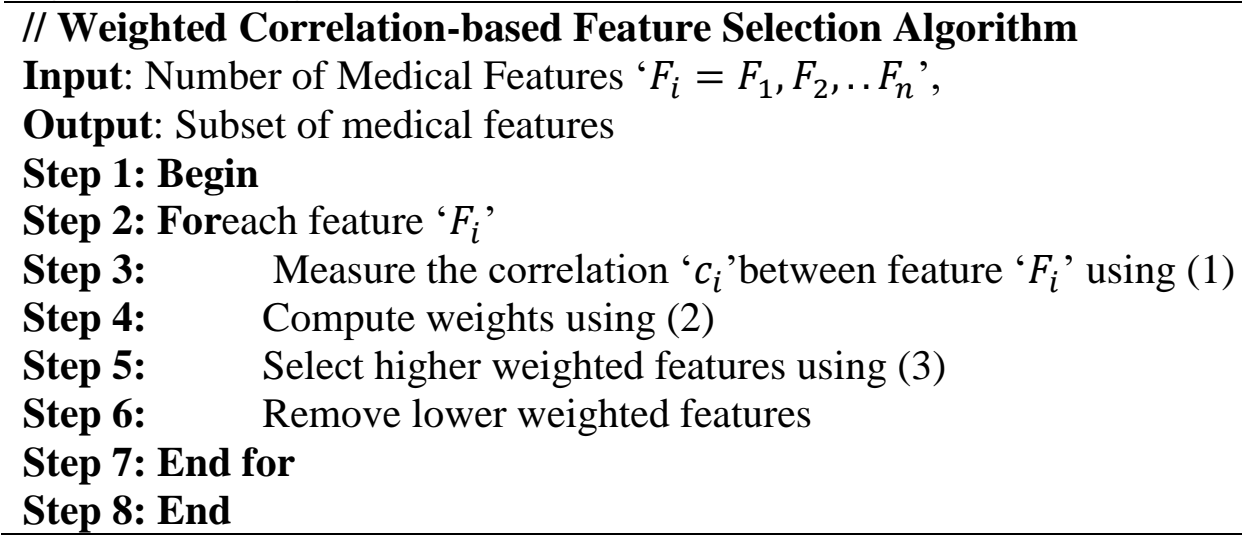

Algorithm 1 depicts the step-by-step processusedbyWC-FS to select significant medical features for improving the performance of brain tumor classification. At first, the medical data is taken as input for brain tumor disease diagnosis. The correlation coefficient all medical feature in the database is measured. Further, the weight values of all medical features are assigned depending on their measured correlation values. From that, the maximum weight features are chosen to perform the classification process. Besides, the minimum weight features are removed from the database. This in turns, the time required to identify the brain tumor disease is highly reduced. With the selected features of medical data, classification is carried out in the next section for identifying brain tumor diseases.

\subsection{Iterative Bayesian Multivariate Deep Neural Network Classifier}

After performing feature selection, the classification is performed using IBMDNN classifier in proposed WCFS-IBMDNL technique. The IBMDNN technique is applied to improve the performance of brain tumor classification. An IBMDNN classifier is developed by combining BMLR and IRLS in the deep neural network. The IBMDNN classifier is a feedforward network where each layer uses output from the previous layer as input. The IBMDNN technique iteratively performs classification using multiple layers where the error rate in the output classification is minimized. The design of an IBMDNN classifier is based on the neural network of the human brain. The IBMDNN classifier uses multiple hidden layers to analyze medical data, which improves the diagnosis rate of brain tumors with lower time complexity.

In contrast to conventional techniques, BMLR is employed in the hidden layer as it helps the IBMDNN classifier to accurately find the relationship between input patient data and disease symptoms in a minimal amount of time. Additionally, IRLS is used in the IBMDNN classifier (in contrast to existing approaches), with the goal of reducing the error rate during brain tumor classification. With the application of BMLR and IRLS, IBMDNN accurately classifies input patient data as normal or abnormal in a minimal amount of time. The IBMDNN classifier process is shown below. 


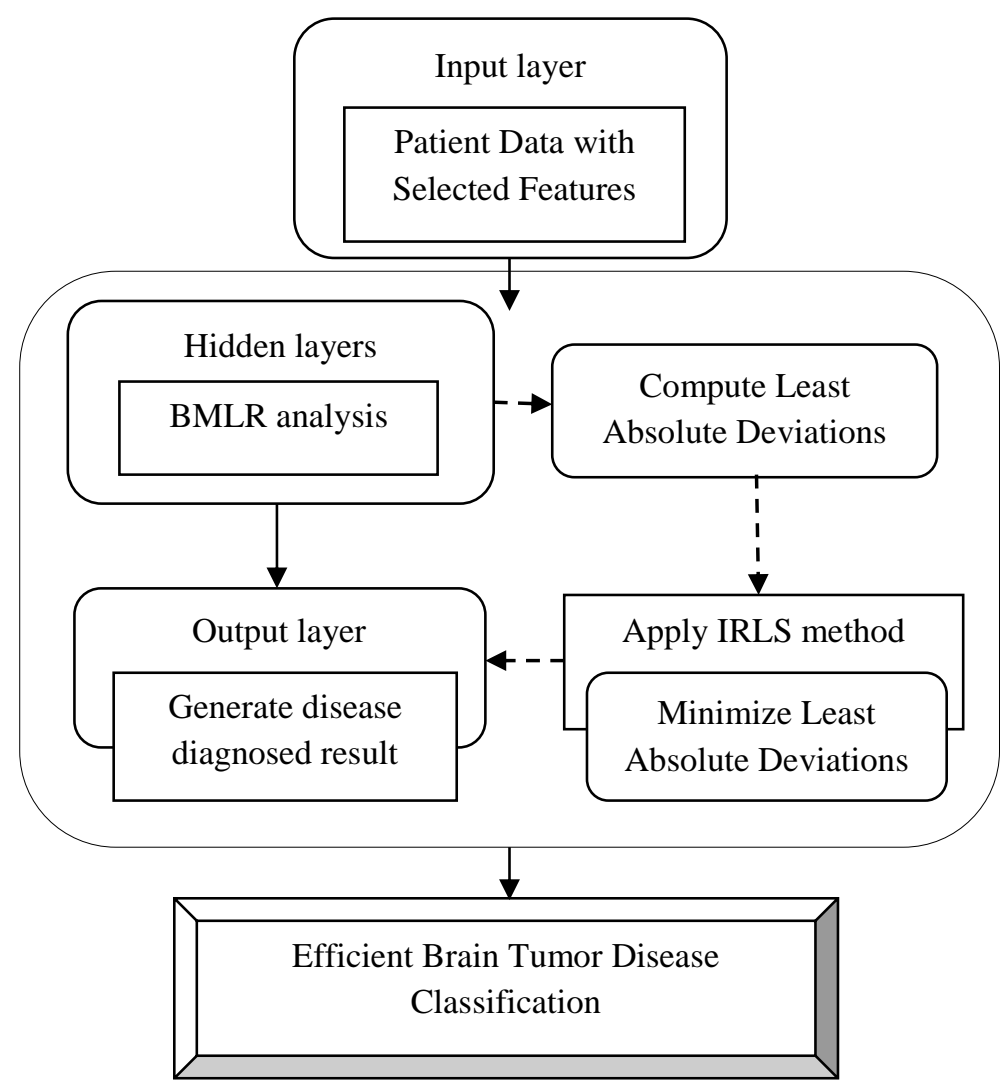

Figure 4. IBMDNN Classifier Process

Algorithm 4showsthe IBMDNN classifier process that is used to accurately detect brain tumors at an early stage in a minimal amount of time. As shown in Algorithm 2, the IBMDNN classifier initializes a neural network with random weights. The input layer uses medical attributes of the patient data as input and sends it to hidden layers. The hidden layers in the IBMDNN classifier analyze the medical attributes of patient data usingBMLR and then sendthe classification results to the output layer. Next, the IBMDNN classifier measures the least absolute deviation of each trained patient data. Subsequently,theleast absolute deviations of brain tumor diagnosis using the IBMDNN classifier are minimized using the IRLS method by updating changed weights with respect totheleast absolute deviation (i.e., error rate). The IBMDNN classifier processis repeated until the error value is small. Finally, the output layer in the IBMDNN classifier provides the diagnostic result.TheIBMDNN classifier provides improvedclassification performance for the diagnosis of brain tumors.

Let us consider a medical dataset ' $M D$ ' comprised of many sets of patient medical data denoted as ' $M D_{i}=\left\{P_{1}, P_{2}, P_{3, \ldots}, P_{N}\right\}$ ', with a selected subset of features $F^{*}=f_{1}, f_{2}, \ldots f_{n}$. ' $N$ ' represents the total number of patient datain a given dataset. In order to classify the patients as normal or abnormal, the IBMDNN classifier is used. TheIBMDNN classifier takes a number of sets of patient medical data ' $P_{i}$ ' and the chosen optimal features ' $F^{*}$ ' as input. Neuronal activity in the input layer ' $(u)$ ' is expressed as:

$$
u(t)=\sum_{i=1}^{N} P_{i} \delta_{u v}+a_{j}
$$

In equation (4), the input layer ' $u$ ' combines the input patient data ' $P_{i}$ ' with weights ' $\delta_{u v}$ ' and bias term ' $a_{j}$ ' ' $u(t)$ ' indicates neuronalactivity in the input layer at a time ' $t$ ' and ' $\delta_{X Y}$ ' refers to weights between the input and hidden layer. After receiving a patient's medical data, the input layer forwardsthe data to the hidden layer. The hidden layers in the IBMDNN 
classifier useBMLR analysis to derive a diagnosis based on a patient's medical data.Neuronal activity in hidden layers ' $v_{i}(t)$ ' is obtained from the following equation:

$$
v_{i}(t)=\alpha \sum_{i=1}^{N} u(t) \delta_{v_{i}}
$$

In equation (5), ' $u(t)$ ' denotes neuronal activity in the input layer, ' $\delta_{v_{i}}$ ' refers to the weight in hidden layers, and ' $\alpha$ ' is an activation function. ' $v_{i}(t)$ ' represents neuronal activity in hidden layers at a time ' $t$ '. The IBMDNN classifier utilizes BMLR analysis in an activation function used in hidden layers that assists to analyze medical features of an input patient. The BMLR is a Bayesian approach of multivariate linear regression. The BMLR identifies the relationship between the selected medical features ' $f_{1}, f_{2}, \ldots f_{n}$ ' of a patient and disease symptoms using Bayesian theorem. The output of activation function is obtained using the equation below:

$$
\alpha=\beta_{1} f_{1}+\beta_{2} f_{2}+. .+\beta_{n} f_{n}
$$

In equation (6), ' $f_{1}, f_{2}, \ldots f_{N}$ ' represents the selected significant medical features of patient data whereas ' $\beta_{1}, \beta_{2}, \ldots \beta_{n}$ ' denotes brain tumor symptoms. The IBMDNN classifier performs BMLR analysis with the aid of Bayesian theorem that finds the probability of disease occurence that is dependent on prior knowledge of conditions (i.e., disease symptoms). Thus, the activation function in the IBMDNN classifier returns as output when the medical features of the input patient data is matched with known conditions. Otherwise, the activation function returns as output when the medical features of patient data are not matched with known conditions. The output of the deep neural network hidden layer is evaluated using the equation below:

$$
v_{i}(t)=\left\{\begin{array}{lr}
\text { if }\left(\left(f_{1}, f_{2}, \ldots f_{N}\right)==\left(\beta_{1}, \beta_{2}, \ldots \beta_{n}\right)\right), & \text { Return } 1 \\
\text { otherwise, } & \text { Return } 0
\end{array}\right.
$$

In equation (7), ' $f_{1}, f_{2}, \ldots f_{N}$ ' refers to patient medical attributes whereas ' $\beta_{1}, \beta_{2}, \ldots \beta_{n}$ ' indicates prior knowledge of conditions (i.e., brain tumor symptoms). The results obtained from the last hidden layer arethen forwarded to the output layer. Hence, neuronal activity in the output layer ' $w(t)$ ' at a time ' $t$ ' is determined as:

$$
w(t)=\delta_{v w} v_{i}(t)
$$

In equation (8), ' $\delta_{v w}$ ' denotes the weight between the hidden and output layer.The brain tumor disease diagnosis results ' $w(t)$ ' at the time ' $t$ ' is estimated as,

$$
w(t)=\left\{\begin{array}{l}
1, P_{i} \text { is abnoraml } \\
0, \quad P_{i} \text { is normal }
\end{array}\right.
$$

In equation (9), ' $w(t)=1$ ' represents abnormal input patient medical data ' $P_{i}$ ' whereas ' $w(t)=0$ ' inidcates that the patient ' $P_{i}$ ' is normal. Inequation (9), the IBMDNN classifier classifies the patient as normal or abnormal.

After obtaining the classification result, the IBMDNN classifier finds the least absolute deviations to improve the diagnosis rateof brain tumorsas compared to existing approaches. Least absolute deviations (LAD) is termedleast absolute errors (LAE) or the sum of absolute deviations. It is defined as the difference between the actual and desired output. LAD is used in the IBMDNN classifier to evaluate the classification error rate during the process of 
predicting brain tumors. The error rate at time ' $e(t)$ ' is estimated using the equation below in [26].

$$
e(t)=\sum_{i=1}^{N}|(w(t)-\overline{w(t)})|
$$

In equation (10), ' $e(t)$ ' is a measured error rate of brain tumor classification at time ' $(t)$ '. Here, ' $\overline{w(t)}$ ' denotes the actual output whereas ' $w(t)$ ' refers to the obtained output. The weights are updated according to the measured error rate. Then, weights are updated using the equation below:

$$
\begin{gathered}
\delta_{u v}^{\prime}=-\mu \frac{\partial e(t)}{\partial \delta_{u v}} \\
\delta_{v}^{\prime}=-\mu \frac{\partial e(t)}{\partial \delta_{v}} \\
\delta_{v w}^{\prime}=-\mu \frac{\partial e(t)}{\partial \delta_{v w}}
\end{gathered}
$$

From equations (11), (12), and (13), ' $\mu>0$ ' refers to a learning rate. From the above equations, weight changes in input, hidden, and output layers ' $\delta_{u v}^{\prime}$ ', ' $\delta_{v}^{\prime}$ ', ' $\delta_{v w}^{\prime}$ ' areupdated according to the error rate ' $e(t)$ '. Then, the IBMDNN classifier applies the IRLS method with the objective of decreasing the error rate and thereby improving the performance of brain tumor classification with a better diagnosis rate. In the IBMDNN classifier, with the help of objective function, the IRLS method is used to resolve certain optimization problems. Here, objective functions minimize the error rate for effective brain tumor prediction. As a result, the IRLS method decreases the error rate of the IBMDNN classifier using the equation below:

$$
w(t)=\arg \min \sum_{i=1}^{N}|(w(t)-\overline{w(t)})|
$$

An iterative method is used, for which each step reduces the least absolute deviations. The IRLS method at step ' $t+1$ ' involves solving the least absolute deviations problem through updating the weight based on the measured error rate using the equation below:

$$
w(t)=\arg \min \sum_{i=1}^{N} \delta_{v}^{\prime(t)}|(w(t)-\overline{w(t)})|
$$

By using equations (14) and (15), the IBMDNNclassifier finds a minimum error for the accurate classification of brain tumors. The algorithmic steps of the IBMDNN classifier are shown below. 


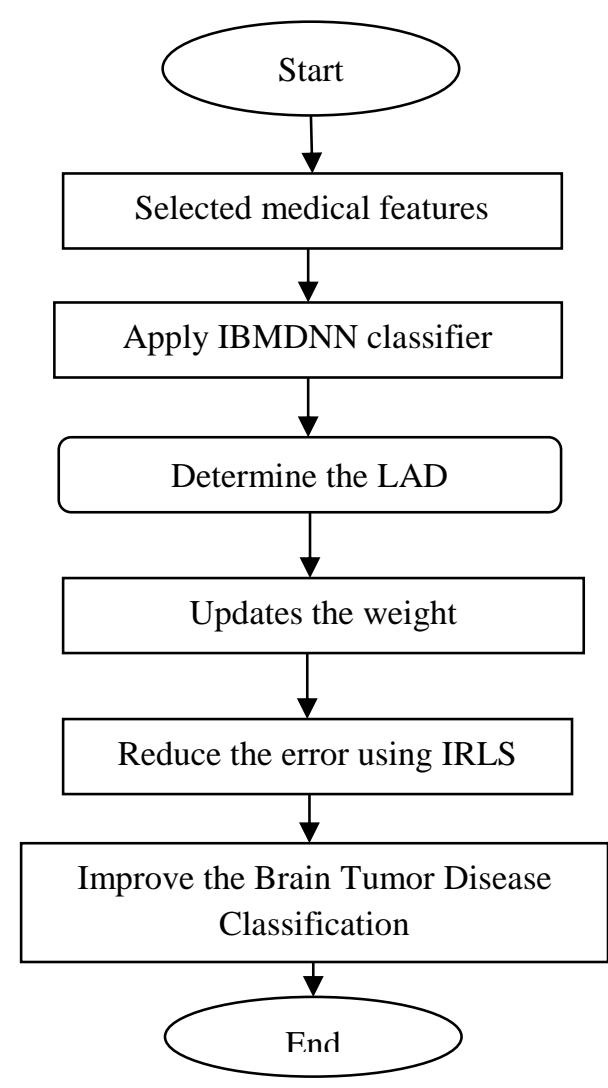

Figure 5 Flow process of IBMDNN Classifier

Figure 5 shows the flow process of IBMDNN Classifier for performing brain tumor disease diagnosis at an early stage. This classification process helps to identify the patient data as normal or abnormal using the medical database.

Algorithm2.IBMDNN Classifier

\section{// IBMDNN Classifier Algorithm}

Input: Medical dataset ' $M D_{i}=\left\{P_{1}, P_{2}, P_{3, \ldots}, P_{N}\right\}$ ' with a selected subset of features ' $F^{*}=f_{1}, f_{2}, \ldots f_{n}$ '

Output: Enhanced DDR with minimal time

Step 1: Begin

Step 2: Randomly initialize neural network with random weights

Step 3: For patient medical data ' $P_{i} \in M D$ '

Step 4: While (' $e(t)$ ' is low) do

Step 5: $\quad$ Input layer ' $u(t)$ ' takes ' $P_{i}$ ' and sends it to be hidden layers using (4)

Step 6: $\quad$ Hidden layers ' $w(t)$ ' deeply analyze medical attributes of ' $P_{i}$ ' using (5)

Step 7: $\quad$ Output layer ' $w(t)$ ' returns diagnosed result using (8)

Step 8: $\quad$ Measure error rate ' $e(t)$ ' using (10)

Step 9: $\quad$ Update weights ' $\delta_{u v}^{\prime}$ ', ' $\delta_{v}^{\prime}$ ', ' $\delta_{v w}^{\prime}$ ' using (11), (12), and (13)

Step 10: $\quad$ Apply the IRLS method to minimize ' $e(t)$ ' using (14) and (15)

Step 11: $\quad$ End while

Step 12: $\quad$ If $(w(t)=1)$ then

Step 13: $\quad$ Classify ' $P_{i}$ ' as abnormal

Step 14: $\quad$ Else

Step 15: $\quad$ Classify ' $P_{i}$ ' as normal 


\section{Step 16:End If}

Step 17: End for

Step 18: End

Algorithm 2 shows the step-by-step process of the IBMDNN classifier for enhanced performance of classification for diagnosing brain tumors at an early stage. With the support of Algorithm 2, the IBMDNN classifier efficiently classifies the patient as normal or abnormal with a higher diagnosis rate. With the aid of multiple hidden layers, the medical features are analyzed with disease symptoms and produce the classification results at the output layer. In addition, the error rate is calculated for output results, and it is reduced using the IRLS method. The WCFS-IBMDNL technique performs brain tumor disease classification as compared to existing approaches, in terms of disease diagnosisrate, DDT, and FAR.

\section{EXPERIMENTAL SETTINGS}

The WCFS-IBMDNL technique is implemented in Java language using the Epileptic Seizure Recognition Dataset [24] to determine its performance. The Epileptic Seizure Recognition Datasetwasobtained from the UCI Machine Learning Repository [25]. This dataset consists of five folders, with each folder containing 100 files and patient medical data. Each file contains 23.6 seconds of brain activity of each patient. Each data point corresponds to the value of the EEG recording at a distinct point in time where data points are considered to biomedical features. Thus, this dataset consists of 500 sets of patient medical data in which each patient has 4,097 data points consisting of 23.5 seconds. Every set of 4,097 data points are split into 23 parts. Each set of patient medical data is comprised of 178 data points consisting of 1 second. The last column signifies the class label. The dataset has 11,500 instances. It is a pre-processed and re-structured version of more generally used dataset. The dataset has the characteristics of multivariate and time-series. It includes 179 attributes, and it has the integer and real characteristic.

To conduct the experimental evaluation, the WCFS-IBMDNL technique takes a different number of sets of patient medical data from the Epileptic Seizure Recognition Dataset. The WCFS-IBMDNL technique enhances the disease diagnosis rate (DDR) and reduces disease diagnosis time (DDT)of the Epileptic Seizure Recognition Dataset as compared to existing approaches. Based on the objective of the proposed WCFS-IBMDNL technique, the performance metrics (i.e., parameters) are chosen to analyze the effectiveness of the proposed technique. The primary objective of WCFS-IBMDNL technique is to improve the early-stage of brain tumor diagnosis with minimum time and FAR. Therefore, experimental results generated by the WCFS-IBMDNL technique are estimated in terms of disease diagnosis rate, DDT, and FAR and are compared with the Ensemble classifier [1], SVM classifier [2] and MRBF-MPSO-SVM [16]. Besides, the cross-validation model is used to evaluate the better performance of WCFS-IBMDNL technique. Here, the Holdout cross-validation method is employed to separate the input dataset into two sets such as training set and the testing set. Holdout cross-validation is a simple kind of cross-validation method.

\section{RESULTS AND DISCUSSION}

In this section, the results of the analysis of the WCFS-IBMDNL technique are presented. The effectiveness of the WCFS-IBMDNL technique is analyzed with the help of graphs using the parameters shown below.

\subsection{Experimental Result Analysis of Disease Diagnosis Rate (DDR)}

The Disease DiagnosisRate (' $D D R$ ') is the ratio of a number of patients correctly classified as normal or abnormal to the total number of patient data experimentally evaluated. The DDR is evaluated in terms of percentages (\%) according to the following equation: 


$$
D P R=\frac{X_{C C}}{N} * 100
$$

Equation (16) measuresthediagnosis rate of brain tumors. Here, ' $N$ ' refers to the total number of sets of patient medical data employed in experimental work, whereas ' $X_{C C}$ ' indicates the number of sets of patient data that are classified as normal or abnormal.

\section{Sample calculation:}

* Ensemble classifier: the number of patients classified is 40and the total number of patients used in the experimental work is 50. TheDDRis measured as:

$$
D D R=\frac{40}{50} * 100=80 \%
$$

* SVM classifier: the number of patients accurately classified is 38 and the total number of patients utilized in the experimental evaluation is 50. The DDR is estimated as:

$$
D D R=\frac{38}{50} * 100=76 \%
$$

* MRBF-MPSO-SVM: the number of the number of patients classified is 42 and the total number of patients used for experimental evaluation is 50. The DDR is determined as:

$$
D D R=\frac{42}{50} * 100=84 \%
$$

* WCFS-IBMDNL Technique: the number of patients correctly classified is 48 and the total number of patients used in the experimental process is 50. Then DDR is determined as:

$$
D D R=\frac{48}{50} * 100=96 \%
$$

The WCFS-IBMDNL technique is implemented in Java language by considering various numbers of patients in the range of 50-500 from the Epileptic Seizure Recognition Dataset to estimate the diagnosis rate of brain tumors. When considering 300 sets of patient medical data, the WCFS-IBMDNL technique has a93\% DDR whereas conventional Ensemble classifier [1] and SVM classifier [2] and MRBF-MPSO-SVM [16] have 87\%, 84\% and 89\% DDRs respectively.

The comparative results of brain tumor DDR are demonstrated on Figure below. 


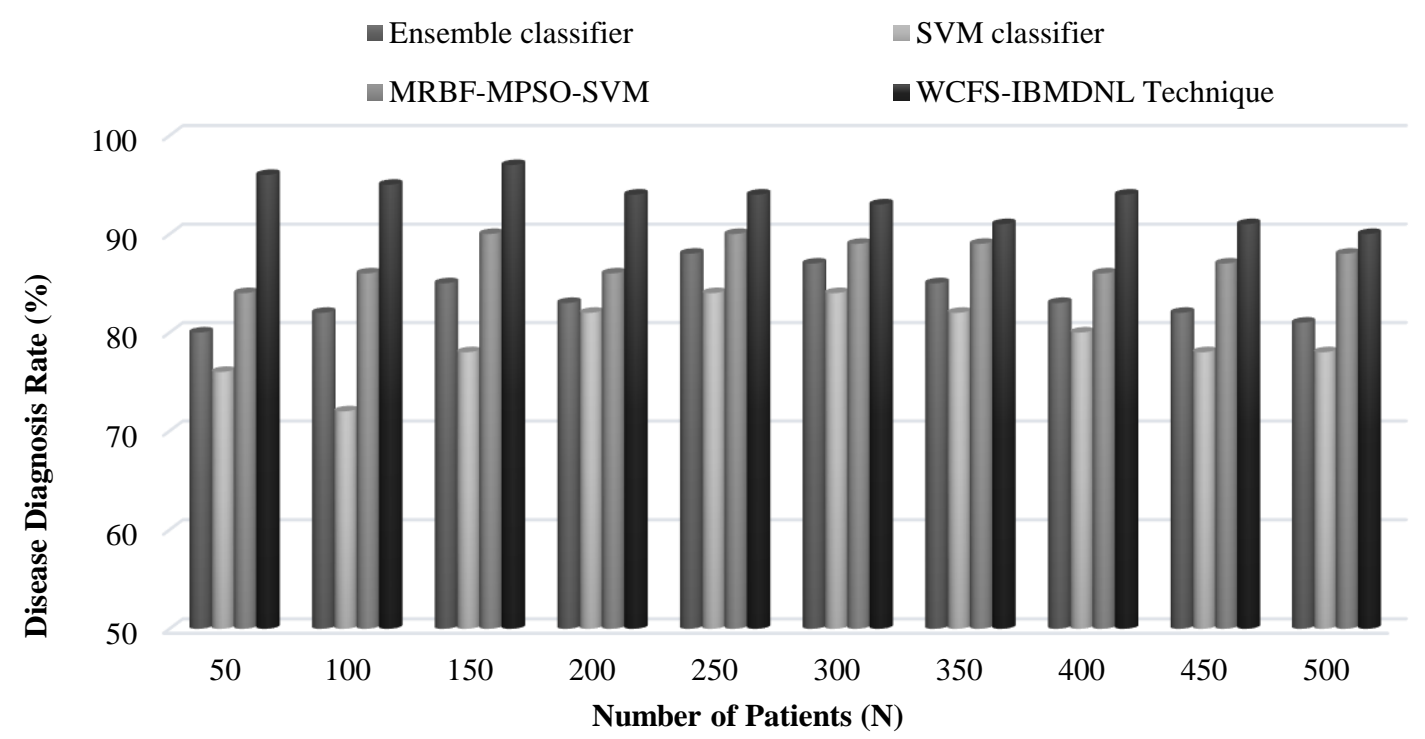

Figure: 6 Comparative Analysis of DDR (\%) vs. Number of Patients (N)

Figure 6 depicts the impact of brain tumor DDR versus a different number of patients using four methods: Ensemble classifier [1], SVM classifier [2], MRBF-MPSO-SVM [16] and WCFS-IBMDNL technique. As illustrated in the Figure 6, the WCFS-IBMDNL technique attains a higher DDR for brain tumors as compared to the existing Ensemble classifier [1], SVM classifier [2], MRBF-MPSO-SVM [16]. This is due to the application of IBMDNN classifier in the WCFS-IBMDNL technique. IBMDNN classifies the selected features that are more significant for performing brain tumor detection. With the classified features, the IBMDNN Classification is performed foraccuratelydiagnosing the patients as normal or abnormal with the help of BMLR and IRLS. This supports the WCFS-IBMDNL technique to improve the ratio of patients correctly classified as normal or abnormal as compared to stateof-the-art approaches. The higher DDR further increases the accuracy of brain tumor disease diagnosis. WCFS-IBMDNL technique enhances the diagnosis rate of brain tumors by $12 \%$, $18 \%$ and $7 \%$ when compared to the Ensemble classifier [1], the SVM classifier [2] and MRBFMPSO-SVM [16] respectively.

\subsection{Experimental Result Analysis of Disease Diagnosis Time (DDT)}

The DDT computes the amount of time needed to discover the presence of a brain tumor through classification. The DDT is estimated in terms of milliseconds (ms) as shown in the equation below:

$$
D D T=N * t(C S P)
$$

Equation (17) determinesthetime required to diagnosea brain tumor. Here, ' $(N)$ ' denotes a number ofsets of patient medical data and ' $t(C S P)$ ' represents the time utilized for classifying the medical data of a single patient.

\section{Sample calculation:}

* Ensemble classifier: the time used to classifyasingle patient's medical data as normal or abnormal is $0.28 \mathrm{~ms}$ and the total number of patients is 50 . Then, the disease diagnosis time is measured as,

$$
D D T=0.28 * 50=14 \mathrm{~ms}
$$

* SVM classifier: the time needed for classifying medical data of a single patient is 0.35 $\mathrm{ms}$ and the total number of patients is 25 . Then, DDT is computed as, 


$$
D D T=0.35 * 50=18 \mathrm{~ms}
$$

MRBF-MPSO-SVM: the time consumed to classify the single patient's medical data as normal or abnormal is $0.26 \mathrm{~ms}$ and the total number of patients is 50 . Then, the disease diagnosis time is determined as,

$$
D D T=0.26 * 50=13 \mathrm{~ms}
$$

* WCFS-IBMDNL Technique: the time required for classifying single patient data is $0.21 \mathrm{~ms}$ and the total number of patients is 50 . Then, DDT is evaluated as,

$$
D D T=0.21 * 50=11 \mathrm{~ms}
$$

To estimate the DDT involved during brain tumor diagnosis, the WCFS-IBMDNL Techniqueis implemented with diverse numbers of patients in the range of 50-500. When employing 350 sets of patient's medical data, the WCFS-IBMDNL Technique takes $35 \mathrm{~ms}$ DDT whereas the state-of-the-art approaches Ensemble classifier [1], SVM classifier [2] and MRBF-MPSO-SVM [16] take $46 \mathrm{~ms}, 60 \mathrm{~ms}$ and $39 \mathrm{~ms}$, respectively.

The results of brain tumor DDT versus a various number of patients using Ensemble classifier [1], SVM classifier [2], MRBF-MPSO-SVM [16] and the WCFS-IBMDNL Technique. As shown in the Figure 7, the WCFS-IBMDNL Technique takes minimal amount of time for finding occurrences of brain tumors as compared to Ensemble classifier [1], SVM classifier [2] and MRBF-MPSO-SVM [16]. The comparative results of brain tumor DDT are shownon figure below.

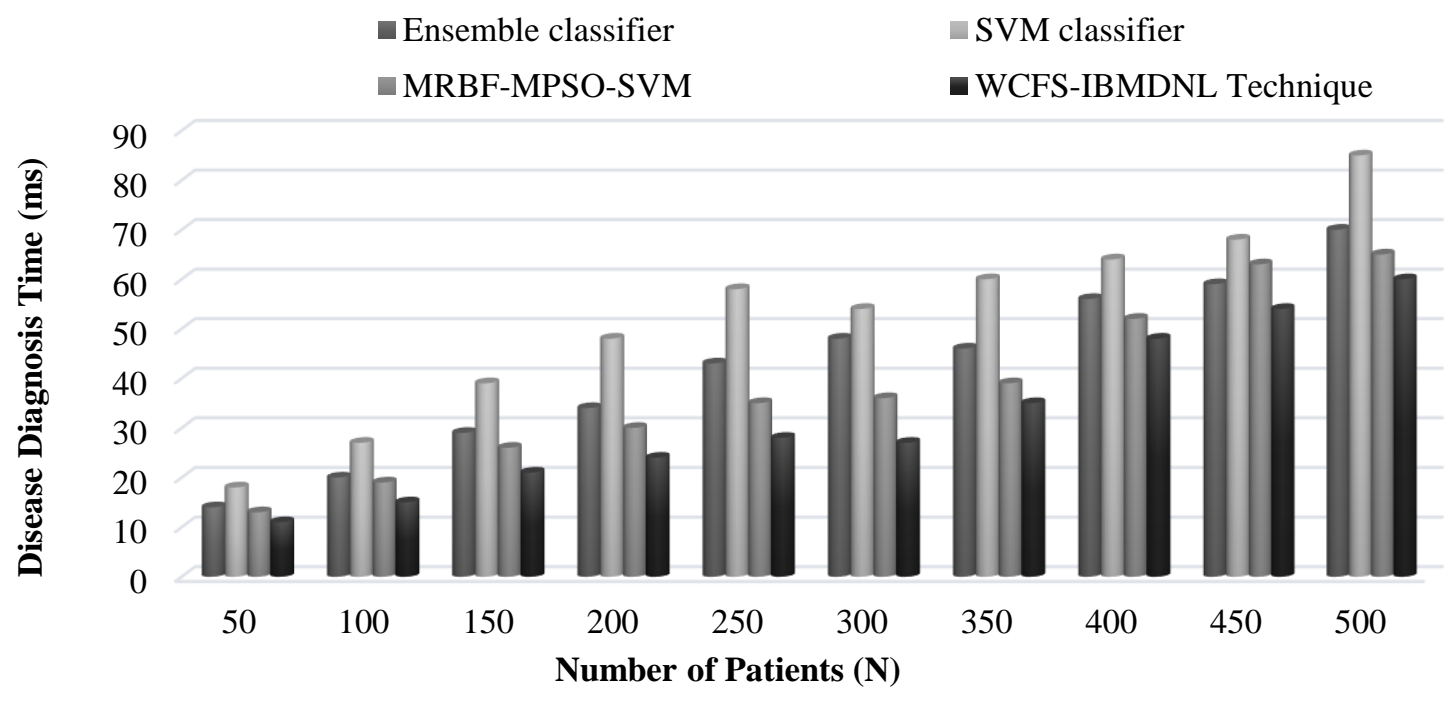

Figure: 7 Comparative Analysis of DDT (ms) vs. Number of Patients (N)

Figure 7 shows the impact of brain tumor DDT versus a different number of patients using four methods: Ensemble classifier [1], SVM classifier [2], MRBF-MPSO-SVM [16] and WCFS-IBMDNL technique. Let us consider the 150 number of patients, DDT using WCFSIBMDNL is $21 \mathrm{~ms}$ whereas the existing Ensemble classifier [1], SVM classifier [2] and MRBFMPSO-SVM [16] acquires DDT of $29 \mathrm{~ms}, 39 \mathrm{~ms}$, and $26 \mathrm{~ms}$ respectively. This is due to the application of WC-FS and the IBMDNN Classifier in the WCFS-IBMDNL Technique in contrast to state-of-the-art approaches. Through the WC-FS processes, the WCFS-IBMDNL Technique selects a subset of medical attributes in a lower amount of time for effective brain tumor classification. Then, IBMDNN Classifier classifies a patient as normal or abnormal in a minimal amount of time. This assists the WCFS-IBMDNL Technique to minimize the amount 
of time needed to discover the presence of brain tumors as compared to existing approaches. WCFS-IBMDNL Technique reduces the time required to diagnose brain tumor disease by 24 $\%, 40 \%$ and $16 \%$ as compared to Ensemble classifier [1], SVM classifier [2] and MRBFMPSO-SVM [16], respectively.

\subsection{Analysis of False Alarm Rate (FAR)}

The False Alarm Rate ' $(F A R)$ ' is evaluated as the ratio of a number of patient data sets wrongly classified as normal or abnormal to the total number of patient data sets. The FAR is measured in terms of percentages $(\%)$ and shown below:

$$
F A R=\frac{X_{W C}}{N} * 100
$$

From equation (18), the FAR of brain tumor classification is obtained. Here, ' $X_{W C}$ ' denotes the patient data that are incorrectly classified as normal or abnormal.

\section{Sample calculation:}

* Ensemble classifier: number of patients mistakenly classified is 10and the total number of patients is 50 . Then, the FAR is estimated as,

$$
F A R=\frac{10}{50} * 100=20 \%
$$

* SVM classifier: number of patients wrongly classified is 12 and the total number of patients is 50 . Then the FAR is determined as,

$$
F A R=\frac{12}{50} * 100=24 \%
$$

MRBF-MPSO-SVM: number of patients inaccurately classified is 11 and the total number of patients is 50 . Then the FAR is determined as,

$$
F A R=\frac{11}{50} * 100=22 \%
$$

* WCFS-IBMDNL Technique: number of patients inaccurately classified is two and the total number of patients is 50 . Then the FAR is obtained as,

$$
F A R=\frac{2}{50} * 100=4 \%
$$

For measuring the FAR during brain tumor classification, the WCFS-IBMDNL technique is implemented with dissimilar numbers of patients in the range of 50-500. When using 400 sets of patient's medical data, the WCFS-IBMDNL Technique attains $6 \%$ FAR whereas Ensemble classifier [1], SVM classifier [2] and MRBF-MPSO-SVM [16] attain $17 \%$, $20 \%$ and $17 \%$ respectively. 


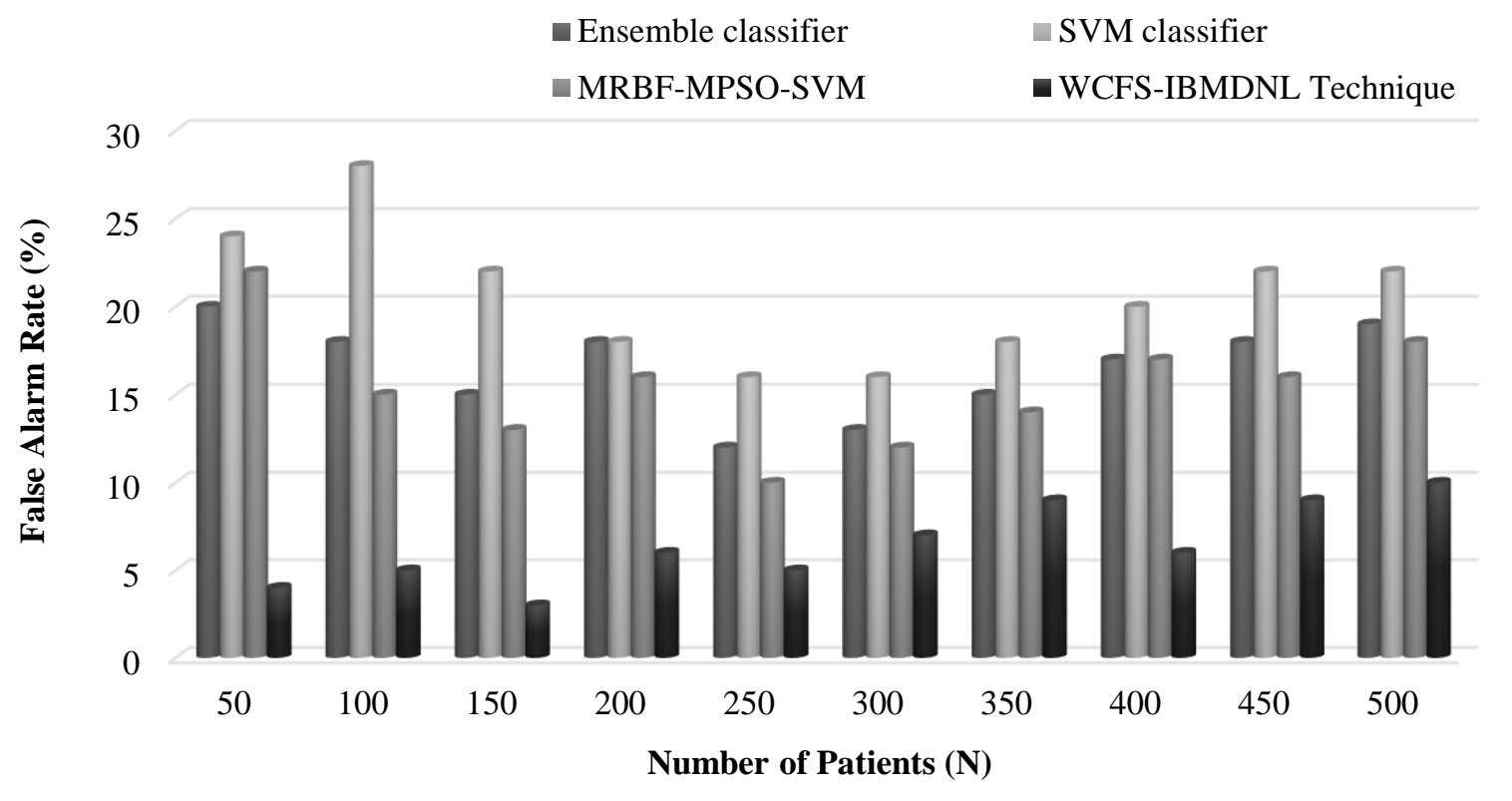

Figure: 8 Comparative result analysis of FAR (\%) Vs. Number of Patients (N)

Figure 8 shows the impact of FAR versus different numbers of patients using Ensemble classifier [1], SVM classifier [2], MRBF-MPSO-SVM [16] and the WCFS-IBMDNL Technique. As shown in figure 8, the WCFS-IBMDNL Technique attains a lower FAR for brain tumor prediction as compared to Ensemble classifier [1], SVM classifier [2] and MRBFMPSO-SVM [16]. The WC-FS and IBMDNN are two classifiers are used to build the WCFSIBMDNL Technique which is efficient when compared to existing approaches. The algorithmic processes of WC-FS assist the WCFS-IBMDNL Technique to choose a subset of medical attributes that are more important for brain tumor disease with lower time complexity. With the aid of the selected subset of medical attributes of a patient, the IBMDNN classifier classifies a patient as normal or abnormal with minimum error. This helps the WCFS-IBMDNL Technique to decrease the ratio of patient data wrongly classified as normal or abnormal as compared to state-of-the-art approaches. By using the proposed model, the error rate of the medical data is reduced while classifying. As a result, the WCFS-IBMDNL Technique reduced the FAR by $61 \%, 68 \%$ and $57 \%$ as compared to Ensemble classifier [1], SVM classifier [2] and MRBF-MPSO-SVM [16], respectively.

\section{CONCLUSION}

An efficient WCFS-IBMDNL technique was designed with the goal of increasing performance of diagnosing brain tumors with minimal FAR. Application of WC-FS and the IBMDNN Classifier achieves the goal of the WCFS-IBMDNL Technique. With the help of the algorithmic processes of WC-FS, the WCFS-IBMDNL technique enhances the performance of feature selection to choose the more relevant subset of medical features in less time. In WCFS, the Pearson correlation coefficient is employed to calculate the correlation between two medical features. Based on the correlation measure, a weight value is assigned in all medical features for detecting relevant classification features of the brain tumor at an early stage. In addition to the algorithmic processes of the IBMDNN, the classifier includes three layers for producing effective classification results with a minimum error. This in turns, WCFSIBMDNL Technique reduces the ratio of patient data sets that are incorrectly classified, which 
provides enhanced brain tumor diagnosing performance as compared to existing approaches. The performance of the WCFS-IBMDNL technique is determined in terms of disease diagnosis rate, DDT, and FAR as compared with two state-of-the-art approaches. The experimental results demonstrated that the WCFS-IBMDNL technique performed better with improvement both in terms of DDR and minimization of DDT for accurate diagnosis of brain tumors. Proposed WCFS-IBMDNL technique is tested with benchmark Epileptic Seizure Recognition Dataset but still, there is a large amount of patient medical data is available which need to be tested with our technique. Future work is also laid in refining the parameter to get more effective and practical classification results. The use of more advanced machine learning algorithms can also enhance brain tumor diagnosis progress further.

\section{REFERENCES}

[1] S Huda, John Yearwood, Herbert F. Jelinek, Mohammad Mehedi Hassan, Giancarlo Fortino, and Michael Buckland, "A Hybrid Feature Selection with Ensemble Classification for Imbalanced Healthcare Data: A Case Study for Brain Tumor Diagnosis", IEEE Access, Volume: 4, Pages 9145 - 9154, 2016.

[2] Ning Wang, Michael R. Lyu "Extracting and Selecting Distinctive EEG Features for Efficient Epileptic Seizure Prediction”, IEEE Journal of Biomedical and Health Informatics, Volume 19, Issue 5, Pages 1648 - 1659, 2015.

[3] J Sachdeva, Jainy, Kumar, Vinod, Gupta, Indra, Khandelwal, Niranjan, Ahuja, Chirag, “A package-SFERCB- "Segmentation, feature extraction, reduction and classification analysis by both SVM and ANN for brain tumors", Applied Soft Computing, Pages 1-16, 2016.

[4] S Tortajada, EliesFuster-Garcia, Javier Vicente, Pieter Wesseling, Franklyn A.Howe, Margarida Julia-Sape, Ana-Paula Candiota, Daniel Monleón, ÀngelMoreno-Torres, JesúsPujol, John R.Griffith, Alan Wright, Andrew C.Peet, Carmen Martínez-Bisbal, Bernardo Celdadl Carle, Arúsdem Montserrat Robles, Juan Miguel García-Gómez, "Incremental Gaussian Discriminant Analysis based on Graybill and Deal weighted combination of estimators for brain tumor diagnosis", Journal of Biomedical Informatics, Elsevier, Volume 44, Issue 4, Pages 677-687, August 2011.

[5] P.Fergus, A.Hussain, David H, D.Al-Jumeily, Khaled Abdel-Aziz, Hani Hamdan, "A machine learning system for automated whole-brain seizure detection", Applied Computing and Informatics, Elsevier, Volume 12, Issue 1, Pages 70-89, January 2016.

[6] Wanpracha Art Chaovalitwongse, Rebecca S. Pottenger, Shouyi Wang, Ya-Ju Fan, Leon D. Iasemidis, "Pattern- and network-based classification techniques for multichannel medical data signals to improve brain diagnosis", IEEE Transactions on Systems, Man, and Cybernetics Part A:Systems and Humans, Volume 41, Issue 5, Pages 977-988, September 2011.

[7] G Reifenberger, Pieter Wesseling, "Molecular diagnostics of brain tumors", Acta Neuropathologica, Springer, Volume 120, Issue 5, Pages 549-551, November 2010.

[8] E Kabir, Siuly, Yanchun Zhang, "Epileptic seizure detection from EEG signals using logistic model trees", Brain Informatics, Springer, Volume 3, Issue 2, Pages 93-100, June 2016.

[9] F Félix. Gonzalez-Navarro, Lluis A. Belanche-Muñoz, Enrique Romero, Alfredo Vellido, Margarida Julia-Sape, CarlesArus, "Feature and model selection with discriminatory 
visualization for diagnostic classification of brain tumors", Neurocomputing, Elsevier, Volume 73, Issues 4-6, Pages 622-632, January 2010.

[10] A.Harshavardhan, Suresh Babu,, T. Venugopal, "Analysis of Feature Extraction Methods for the Classification of Brain Tumor Detection", International Journal of Pure and Applied Mathematics,Volume 117, Issue 7, Pages 147-155, 2017.

[11] Mustafa Serter Uzer, Nihat Yilmaz, and OnurInan, "Feature Selection Method Based on Artificial Bee Colony Algorithm and Support Vector Machines for Medical Datasets Classification", The Scientific World Journal, Hindawi Publishing Corporation, Volume 2013, Pages 1-10, July 2013.

[12] P. Shanthakumar, P. Ganeshkumar, "Performance analysis of classifier for brain tumor detection and diagnosis", Computers and Electrical Engineering, Computers \& Electrical Engineering, Elsevier, Volume 45, Pages 302-311, July 2015.

[13] A. Aarabi, R.Fazel-Rezai, Y.Aghakhani, “A fuzzy rule-based system for epileptic seizure detection in intracranial EEG”, Clinical Neurophysiology, Elsevier, Volume 120, Issue 9, Pages 1648-1657, September 2009.

[14] R Hopfengärtner, Burkhard S.Kasper, Wolfgang Graf, Stephanie Gollwitzer, Gernot Kreiselmeyer, Hermann Stefan, Hajo Hamer, "Automatic seizure detection in long-term scalp EEG using an adaptive thresholding technique: A validation study for clinical routine", Clinical Neurophysiology, Volume 125, Issue 7, Pages 1346-1352, July 2014.

[15]R Hussein, Hamid Palangi, Rabab Ward, Z. Jane Wang, "Epileptic Seizure Detection: A Deep Learning Approach”, Electrical Engineering and Systems Science, Signal Processing, Pages 1-12, 2018.

[16] Y. Yang Li, Xu-Dong Wang, Mei-Lin Luo, Ke Li, Xiao-Feng Yang, and Qi Guo, "Epileptic Seizure Classification of EEGs using Time-Frequency Analysis based Multiscale Radial Basis Functions", IEEE Journal of Biomedical and Health Informatics, Volume 22, Issue 2, Pages 386 - 397, March 2018

[17] S Siuly, Yanchun Zhang, "Medical Big Data: Neurological Diseases Diagnosis through Medical Data Analysis", Data Science and Engineering, Springer, Volume 1, Issue 2, Pages 54-64, June 2016

[18] P. Li, Chandan Karmakar, John Yearwood, Svetha Venkatesh, Marimuthu Palaniswami, Changchun Liu, "Detection of an epileptic seizure based on entropy analysis of short-term EEG”, PLoS ONE, Volume 13, Issue 3, Pages 1-17, 2018

[19] Q. Liu, QiongGucZhaoWu, "Feature selection method based on support vector machine and shape analysis for high-throughput medical data", Computers in Biology and Medicine, Elsevier, Volume 91, Pages 103-111, December 2017

[20] P. Zhou, Xuegang $\mathrm{Hu}$, Peipei Li, Xindong Wu, "Online feature selection for highdimensional class-imbalanced data", Knowledge-Based Systems, Elsevier, Volume 136, Pages 187-199, November 2017

[21] A. Hamza Osman and Hani Moetque Aljahdali, "Diabetes Disease Diagnosis Method based on Feature Extraction using K-SVM", International Journal of Advanced Computer Science and Applications, Volume 8, Issue 1, Pages 236-244, 2017 
[22] F.PatlarAkbulut and Aydın Akan, "Support Vector Machines Combined with Feature Selection for Diabetes Diagnosis", Istanbul University-Journal of Electrical \& Electronics Engineering (IU-JEEE), Volume 17, Issue 1, Pages 3219-3225, 2017

[23] C. Ramalingaswamy, Damodar Reddy Edla, VenkatanareshbabuKuppili, Ramesh Dharavath, "RST-BatMiner: A Fuzzy Rule Miner Integrating Rough Set Feature Selection and Bat Optimization for Detection of Diabetes Disease", Applied Soft Computing, Elsevier, Pages $1-50,2017$

[24] Epileptic Seizure Recognition Dataset.

https://archive.ics.uci.edu/ml/datasets/Epileptic+Seizure+Recognition [Accessed by 25 May 2018]

[25] Machine Learning Repository

https://archive.ics.uci.edu/ml/datasets/Epileptic+Seizure+Recognition. [Accessed by 25 May 2018]

[26] Rahib H. Abiyev and Mohammad Khaleel Sallam Ma'aitaH, "Deep Convolutional Neural Networks for Chest Diseases Detection”, Journal of Healthcare Engineering, Hindawi, Volume 2018, pages 11 . 